\title{
Implementation of diffusion and electrostatic forces to produce a new slip velocity in the multiphase approach to nanofluids
}

\author{
M Mahdavi, M Sharifpur* and JP Meyer \\ ${ }^{*}$ Corresponding author
}

\author{
*Tel: +27 124202448 *Email: mohsen.sharifpur@up.ac.za \\ Department of Mechanical and Aeronautical Engineering, University of Pretoria, \\ Private Bag X20, Hatfield, 0028, Pretoria, South Africa \\ http://dx.doi.org/10.1016/j.powtec.2016.11.032
}

\begin{abstract}
Due to the improvement of heat transfer by nanofluids, an understanding of the interactions between nanoparticles and the base fluid is essential for simulation. The relative or slip velocity between nanoparticles and the base fluid is one of the main factors in choosing the multiphase mixture model approach. In this paper, a new slip velocity is proposed and used to compare the simulation result to the experimental results of natural convective flow in a cavity filled with an alumina nanofluid. Therefore, the ANSYS-Fluent 15.0 software is employed and the new slip velocity is applied as a user-defined function. The new slip velocity is a result of the combination of Brownian and thermophoretic diffusions, lift, buoyancy and centrifugal forces, virtual mass, pressure gradient, Van der Waals attraction and electric double layer repulsion forces. The comparison between these forces and induced drag force will provide the corresponding slip velocity. The simulation results were in good agreement with the flow pattern and heat transfer features of the experimental studies in the literature. It was found that thermophoretic and electrostatic slip mechanisms should essentially be considered in simulations, as well as buoyancy force. The major effects of electrostatic slip velocity are mainly seen in concentration higher than 1 vol.\%, while thermophoresis could not be ignored in any concentration. Therefore, the implemented slip velocity reveals some critical aspects of nanoparticle and base fluid interactions compared to an algebraic velocity.
\end{abstract}

Keywords: Nanofluid, cavity, slip velocity, ANSYS-Fluent, electrostatic forces

\section{Introduction}

The applicability of nano-sized materials suspended in liquids for the enhancement of heat transfer has been a source of much interest in recent years. The improvement of thermal conductivity and the possibility of uniform distribution are considered to be advantages of nanoparticles, especially for small ranges of volume concentrations [1-5]. On the other hand, the negative effects on the mixture's total viscosity may improve or deteriorate the heat transfer, particularly for natural convection [6-9].

Many experimental studies on nanofluids have been conducted in a wide range of flow regimes [10-15], but none of the available theoretical and modelling approaches can fully 
explain the entire phenomena inside the nanofluid. One of the most applicable approaches is the mixture model where there is a continuum of both the nanoparticles and the base fluid. The main interaction between phases in the mixture model occurs by implementing relative velocity. Goodarzi et al. [16], Xuan and Roetzel [17] and Maiga et al. [18] assumed no drift or slip velocity between the nanoparticles and continuous phase, which contradicts the observation of other researchers.

Zero slip velocity supports the idea of homogenous distribution or a lack of particle diffusion inside the base fluid. The abovementioned authors explained the nanofluid mixture properties as the dominant factor to determine the heat transfer features of the flow. Akbarinia and Laur [19], Bianco et al. [20], Rashidi et al. [21] and some more in recent years [22-26] considered centrifugal and gravitational forces as the main reason for the accelerating particle with respect to the base fluid.

Therefore, they discussed the possibility of non-homogeneity of nanoparticle concentration distribution inside the mixture. However, they only employed the default algebraic slip velocity available in the ANSYS-Fluent software without any development for nanoscale application. Buongiorno [27], Hwang et al. [28] and others [29-34] stated that the diffusion flux due to the gradient of concentration and thermophoretic force can be the dominant interaction phenomena between two phases. This idea can be mainly followed by the scale analysis of Buongiorno [27] and Hwang et al. [28], while comparing it to other mechanisms such as gravity, inertia, diffusiophoresis, the magnus effect, fluid drainage, viscosity gradient and non-uniform shear rate. However, it was shown that the influences of slip mechanism between particles and fluid on flow field cannot be ignored.

The literature review shows that, despite the reasonable prediction of the Nusselt number in most of the cases, the results of disturbed flow patterns and concentration distribution could only be reliable when the roles of all the slip mechanisms are considered. Therefore, a new slip velocity equation is developed for the mixture model in this study with the assumption that the resulting drag force is induced by each interaction force.

\section{Description of the numerical approach}

\subsection{Mixture model theory}

The main assumption of the mixture model is based on the validity of the continuum theory in a binary mixture. It can easily be verified by checking the Knudsen number less than 0.1 for nanoparticles inside the liquid, as $K n=\lambda / d_{p}$, where $\lambda$ and $d_{p}$ indicate the mean free path of the liquid phase $(0.3 \mathrm{~nm}$ for water) and nanoparticle mean diameter (normally from 50 to $200 \mathrm{~nm}$ ), respectively. The momentum and energy equations of both phases are combined to form one set of equations with a mixture of thermophysical properties. The mixture continuity, momentum and energy equations for steady conditions are as follows [35]:

$$
\begin{aligned}
& \nabla \cdot\left(\rho_{m} \vec{V}_{m}\right)=0 \\
& \nabla \cdot\left(\rho_{m} \vec{V}_{m} \vec{V}_{m}\right)=-\nabla P_{m}+\nabla \cdot \tau_{m}+\rho_{m} g-\nabla \cdot\left(\frac{\alpha_{p}}{\left(1-\alpha_{p}\right)} \frac{\rho_{p} \rho_{m}}{\rho_{c}} \vec{V}_{p m} \vec{V}_{p m}\right)
\end{aligned}
$$


$\nabla \cdot\left(\rho_{m} \vec{V}_{m} H_{m}\right)=-\nabla \cdot q_{m}-\nabla \cdot\left(\alpha_{p} \rho_{p} \vec{V}_{p m}\left(H_{p}-H_{c}\right)\right)$

where $\alpha_{p}, h_{p}, h_{c}$ and $\vec{V}_{m}$ represent the particle volume fraction, particle enthalpy, fluid enthalpy and mixture velocity respectively. The mixture shear stress $\left(\tau_{m}\right)$ is similar to the single-phase, except that the viscosity of the nanofluid is replaced. The important parameter in the equations is drift velocity $\vec{V}_{p m}$ [35]:

$$
\begin{gathered}
\vec{V}_{p m}=\vec{V}_{p}-\vec{V}_{m} \\
\vec{V}_{m}=\frac{\sum_{k=1}^{2} \alpha_{k} \rho_{k} \vec{V}_{k}}{\rho_{m}}
\end{gathered}
$$

The drift and slip velocity are correlated as [35]:

$$
\vec{V}_{p m}=\vec{V}_{\text {slip }}-\frac{\alpha_{p} \rho_{p}}{\rho_{m}} \vec{V}_{\text {slip }}=\frac{\alpha_{c} \rho_{c}}{\rho_{m}} \vec{V}_{\text {slip }}
$$

The concentration field of nanoparticles is computed from the modified continuity equation for the second phase as follows [35]:

$$
\nabla \cdot\left(\alpha_{p} \vec{V}_{m}\right)=-\nabla \cdot\left(\alpha_{p} \vec{V}_{p m}\right)
$$

\subsection{Developing the slip velocity}

The slip velocity between two phases is induced by the interaction forces. This means that each force can be responsible for a small amount of slip velocity between the particles and the fluid. Therefore, the final form of the slip velocity can be arranged as follows:

$V_{\text {slip }}=V_{\text {Brownian }}+V_{\text {Thermophoesis }}+V_{\text {Virtual-mass }}+V_{\text {Pressure }}+V_{\text {lift }}+V_{\text {Buoyancy-entrifugal }}+V_{V D W}+V_{E D L}$

Equation 8 shows the slip velocity that is induced by Brownian diffusion, thermophoresis, virtual mass, pressure gradient, lift, buoyancy, centrifugal forces, Van der Waals attraction and electrical double-layer repulsion forces.

Buongiorno [27] and Hwang et al. [28] combine Brownian diffusion and thermophoretic forces as follows:

$$
\begin{aligned}
J_{p} & =-\frac{\rho_{p} K_{B} T}{3 \pi \mu_{c} d_{p}} \nabla \alpha_{p}-S_{T} \alpha_{p} \frac{\rho_{p}}{\rho_{c}} \frac{\mu_{c}}{T} \nabla T \\
J_{p} & =\alpha_{p} \rho_{p} \vec{V}_{p m} \\
S_{T} & =0.26 \frac{k_{c}}{k_{p}+2 k_{c}}
\end{aligned}
$$

where $J_{p}$ and $K_{B}$ are Brownian and thermophoresis diffusion flux and the Boltzmann constant, $1.38 \times 10^{-23}$, respectively.

Virtual mass and pressure gradient forces $[35,36]$ are shown as follows: 


$$
\begin{aligned}
& F_{\text {Virtual-mass }}=0.5 \frac{\rho_{c}}{\rho_{p}}\left(\vec{V}_{p} \nabla \vec{V}_{c}-\frac{d \vec{V}_{p}}{d t}\right) \\
& F_{\text {Pressure }}=\frac{\rho_{c}}{\rho_{p}} \vec{V}_{p} \nabla \vec{V}_{c}
\end{aligned}
$$

The final form of the caused slip velocity can easily be calculated by considering the following equation [35]:

$$
\begin{aligned}
& F_{\text {drag }}=\frac{f_{d}}{\tau} \vec{V}_{\text {slip }} \\
& \tau=\frac{\rho_{p} d_{p}^{2}}{18 \mu_{c}}
\end{aligned}
$$

where $\tau$ and $f_{d}$ are the particle relaxation time and drag function based on the particle Reynolds number as:

$$
\begin{aligned}
& f_{d}=1+0.15 \operatorname{Re}_{p}^{0.687} \\
& \operatorname{Re}_{p}=\frac{d_{p} \rho_{c} V_{\text {slip }}}{\mu_{c}}
\end{aligned}
$$

Lift force $[37,38]$ is shown as follows:

$$
\begin{aligned}
& F_{\text {lift }}=C_{L} \frac{\rho_{c}}{\rho_{p}} \alpha_{p}\left(\vec{V}_{p}-\vec{V}_{c}\right) \times\left(\nabla \times \vec{V}_{c}\right) \\
& C_{L}=6.46 \frac{3}{2 \pi \sqrt{\operatorname{Re}_{\omega}}} \\
& \operatorname{Re}_{\omega}=\frac{\rho_{c}\left|\nabla \times \vec{V}_{c}\right| d_{p}^{2}}{\mu_{c}}
\end{aligned}
$$

Slip velocity due to buoyancy and centrifugal forces as used in the default algebraic slip velocity is available in ANSYS-Fluent 15.0:

$$
F_{\text {Buoyancy-centrifugal }}=\frac{\left(\rho_{p}-\rho_{m}\right)}{\rho_{p}}\left(\vec{g}-\left(\vec{V}_{m} . \nabla\right) \vec{V}_{m}-\frac{\partial \vec{V}_{m}}{\partial t}\right)
$$

The Van der Waals attractive force [36] is shown as follows:

$$
\begin{aligned}
& F_{V D W}=-\frac{6}{\pi d_{p}^{3}} \frac{A}{6 d_{p}} \frac{1}{x^{2}(x+1)^{3}(x+2)^{2}} \\
& x=\frac{h}{d}
\end{aligned}
$$

where $A$ and $h$ are the Hamaker constant and the surface-to-surface distance of two approaching particles. The amount of the Hamaker constant is available for some common solid liquid mixtures [36], $4 \times 10^{-20} \mathrm{~J}$ in the case of alumina nanofluid.

Electrical double layer repulsion force [39]:

$$
\begin{array}{llrl}
V_{R} & =\pi d_{p} \varepsilon_{0} \varepsilon_{r} \psi^{2} \exp (-\kappa h) & & \kappa d_{p}<10 \\
V_{R} & =\pi d_{p} \varepsilon_{0} \varepsilon_{r} \psi^{2} \operatorname{Ln}[1-\exp (-\kappa h)] & & \kappa d_{p}>10
\end{array}
$$


$F_{E D L}=-\frac{6}{\pi d_{p}^{3}} \frac{d V_{R}}{d h}$

where $V_{R}$ is the repulsion energy on the surface of a particle. The amount of vacuum and relative permittivity of the medium are $\varepsilon_{0}=8.854 \times 10^{-12} \mathrm{CV}^{-1} \mathrm{~m}^{-1}$ and $\varepsilon_{r}=80$ for water respectively; $\psi$ is the potential on the surface of the electrical double-layer overcharged surface group, which can be approximated by zeta potential on the surface of the diffuse layer. Since the small zeta potential means less repulsion barriers and consequently stronger agglomeration inside the nanofluid, the approaching potential to zero is avoided in all the simulations; $\kappa$ is the Debye-Huckel parameter and is defined as follows [36]:

$\kappa=\sqrt{\frac{2000 F^{2} I_{0}}{\varepsilon_{0} \varepsilon_{,} R T}}$

$F=96485 \mathrm{Cmol}^{-1}, R=8.31 \mathrm{Jmol}^{-1} \mathrm{~K}^{-1}$

where $F$ and $R$ are the Faraday and gas universal constants. The ionic strength is simply calculated by knowing the concentration $\left(\mathrm{c}_{\mathrm{i}}\right)$ and the charge $\left(\mathrm{z}_{\mathrm{i}}\right)$ of the species. With the assumption of the presence of only two ions $\left(\mathrm{H}^{+}\right.$and $\left.\mathrm{OH}^{-}\right)$in the mixture, we have the following [36]:

$I_{0}=\frac{1}{2} \sum_{i} c_{i} z_{i}=\frac{1}{2} \begin{cases}10^{-(14-P H)} \quad P H>7 \\ 10^{-P H} \quad P H \leq 7\end{cases}$

It is noted that the inverse amount of the Debye-Huckel parameter can be scaled as the Debye length or the thickness of the electrical double layer $\left(L_{D}=\kappa^{-1} m\right)$. This length can be used as a cut-off distance to avoid the overlapping of a diffuse layer of nanoparticles. This means that the attractive and repulsive electrostatic forces are only active in the program until the surface-to-surface distance of the particles is higher than $2 L_{D}$.

\subsection{The mixture thermophysical properties and simulation considerations}

Due to the buoyancy effects of the base fluid in natural convection, a high accurate density correlation by curve fitting, found in the American Society of Heating, Refrigerating and Airconditioning Engineers (ASHRAE) Handbook [40] for water, was employed in all the equations as follows:

$$
\rho_{\mathrm{c}}=0.0000152322 \mathrm{~T}^{3}-0.0183891979 \mathrm{~T}^{2}+6.6564073561 \mathrm{~T}+243.4039040229
$$

The other thermophysical and transport properties of the nanofluid mixture are presented as follows [41]:

$$
\begin{aligned}
& \rho_{m}=\sum_{k=1}^{2} \alpha_{k} \rho_{k} \\
& C_{P_{m}}=\frac{\sum_{k=1}^{2} \alpha_{k} \rho_{k} C_{P_{k}}}{\rho_{m}}
\end{aligned}
$$




$$
\begin{aligned}
\frac{k_{m}}{k_{c}} & =1+4.4 \operatorname{Re}^{0.4} \operatorname{Pr}^{0.66}\left(\frac{T}{T_{f r}}\right)^{10}\left(\frac{k_{p}}{k_{c}}\right) \alpha_{p}^{0.66} \\
\operatorname{Re} & =\frac{\rho_{c} u_{B} d_{p}}{\mu_{c}} \\
u_{B} & =\frac{2 K_{B} T}{\pi \mu_{c} d_{p}^{2}} \\
\frac{\mu_{m}}{\mu_{c}} & =\frac{1}{1-34.87\left(d_{p} / d_{c}\right)^{-0.3} \alpha_{p}^{1.03}}
\end{aligned}
$$

where $T_{f r}$ and $d_{c}$ are the water freezing temperature and molecule diameter as $0.3 \mathrm{~nm}$. ANSYS-Fluent 15.0 was employed to simulate the nanofluid-filled cavity. All the above mentioned slip mechanisms were implemented in the mixture model as a new slip velocity equation through the user-defined function (UDF). The UDF was divided into three sections: adjust function to define the gradient of concentration, slip velocity function and mixture thermophysical properties. A two-dimensional cavity with a uniformly structured fine mesh was used.

Three different references are employed to validate the proposed model in this study. A highresolution numerical grid is of course needed in each case because of the thin boundary layer of natural convection in the vicinity of the walls. The streamlines are compared to the study of $\mathrm{Wu}$ et al. [42]. The heat transfer characteristics of the nanofluid are evaluated by Ho et al. [43].

\section{Results and discussion}

For validation purposes, the results of flow patterns and isotherms are compared to the experimental study of $\mathrm{Wu}$ et al. [42].

It is noted that $\mathrm{Wu}$ et al. [42] mentioned a 5\% uncertainty in terms of the temperature measurement and gradient. The good agreement between experimental observation and simulation findings for this case can be a validation remark to properly present the flow pattern and temperature distribution for nanofluids.

The proposed slip velocity's validity is evaluated by comparing it to the experimental Nusselt number as measured by Ho et al. [43] for the alumina nanofluid in Figure 2. The Rayleigh number is defined based on the average temperature of the hot and the cold walls as $g \rho C_{p} \beta\left(T_{h}-T_{c}\right) L^{3} / v k$. The uncertainty in the study of Ho et al. [43] was indicated as 7.0 to $26.9 \%$ for the average Nusselt number and 5.7 to $23.4 \%$ for the Rayleigh number. As shown in Figure 2, the Nusselt number calculated by both the algebraic and the proposed slip velocity are in the range of the $5 \%$ error bar added to the experimental measurment in most of the cases. The abilities of new slip velocity compared to the algebraic velocity can mainly be seen in Figure 2b, which presents the results for the higher concentration of 2 and 3\%. The Nusselt number, which is estimated by the proposed slip velocity, indicates an improvement of 2 to $7 \%$ over conventional velocity. A proportional magnitude analysis of the slip velocity produced by each force can explain this difference (see Table 1). 
The values in Table 1 are average in the computational domain and can change by one order of magnitude due to the different thermal boundary conditions. It is seen that the slip mechanism can be safely neglected in the simulations due to the virtual mass, gradient of pressure and lift. The diffusion force by Brownian motion has shown low impacts, while thermophoretic and gravity slip velocity play a significant role in almost all the cases. As expected, attractive and repulsive electrostatic slip mechanisms are weak for lower than $1 \%$ volume concentration due to the large surface-to-surface distances between the particles. In addition, the Van der Waals attraction only becomes comparable to repulsion in a high volume fraction of $3 \%$.

\subsection{Prediction of fluid and particle flow patterns}

The distribution of the base fluid velocity in the Y-direction and temperature are presented non-dimensionally in figures 3 and 4 . The non-dimensional parameters are $\left(T-T_{c}\right) /\left(T_{h}-T_{c}\right)$

and $V_{y} / \sqrt{g \beta\left(T_{h}-T_{c}\right) L}$. The temperature profiles are more disturbed with the increase in the volume fraction of nanoparticles, while there are almost no changes in the fluid velocity of the $3 \%$ alumina nanofluid with algebraic slip velocity (see Figure 3a). On the other hand, the effects of electrostatic and thermophoretic slip velocity are clearly shown in 2.0 and 3.0 vol.\% in figures $3 \mathrm{~b}$ and $3 \mathrm{~d}$ and figures $4 \mathrm{~b}$ and $4 \mathrm{~d}$. Due to the weak effects of these forces in 0.1 vol. $\%$, not many changes are observed in the nanofluid velocity and temperature profiles as opposed to the water-filled cavity. In addition, the assumption of algebraic slip velocity shows no impacts on the base fluid velocity and temperature profiles for less than 2 vol. $\%$.

Because of the only effect of gravity in algebraic slip velocity, the nanoparticles are mainly scattered at the top (lower concentration) and bottom (higher concentration) (see figures 5a, $5 \mathrm{c}$ and $5 \mathrm{e}$ ). The good dispersion of nanoparticles with the proposed slip velocity is is shown in figures $5 \mathrm{~b}, 5 \mathrm{~d}$ and $5 \mathrm{f}$. In particular, the particle distribution is also observed in the vicinity of the hot and the cold walls due to the thermophoretic slip velocity in Figure 5b. In fact, Figure $5 \mathrm{~b}$ shows the competition among attractive and repulsive electrostatic, thermophoretic and gravitational forces.

A higher mass flow rate is predicted in a cavity filled with alumina nanofluid by implementing the proposed slip velocity in Figure 6. It is observed that the main slip mechanism is gravity in a $1 \%$ nanoparticle concentration, since both the nanoparticle streams that are estimated by algebraic and proposed slip velocities are similar. The increase in particles' mass flow rate, with all the affecting forces considered, is clearly expected in 2.0 and 3.0 vol.\% in both the X- and Y-directions, as shown in figures $6 \mathrm{a}, 6 \mathrm{~b}, 6 \mathrm{c}$ and $6 \mathrm{~d}$. The particle stream boundary layer in the Y-direction is almost twice as thick as that in the $\mathrm{X}$ direction, which is due to the additional effect of gravity in the Y-direction only.

The vector of mean velocity in the cavity is shown in Figure 7. It is coloured by the magnitude of the velocity. The order of the velocity is found $10^{-3}$ which only occurs close to the hot wall. The trend of the vectors clearly present the circulation inside the cavity.

\section{Conclusion}

The heat transfer enhancement applications of nanofluids in recent years was the main motivation of this study, which aimed to investigate the interactions between particles and liquids in more detail. Therefore, a new correlation for the slip velocity between 
nanoparticles and the base fluid was formulated in the mixture model. It was replaced with the default algebraic slip velocity available in ANSYS-Fluent 15.0 software through the UDF. The results of laminar natural convection were in good agreement with the experimental findings in a cavity filled with alumina nanofluid. It was found that the gravitational and thermophoretic slip mechanisms were the only dominant slip velocities for less than 1 vol.\%.

However, the competition between gravitational, thermophoretic and electrostatic slip mechanisms became important with an increase in nanoparticle concentration. The Van der Waals attraction slip velocity was found to be comparable with the electric double layer repulsion when the volume concentration was increased by 2.0 and even $3.0 \%$. The Brownian diffusion has shown weak impacts in all the simulations. The lift, virtual mass and pressure gradient slip mechanisms can be safely ignored in any range of volume concentrations for natural convection. The nanoparticles were predicted to be well dispersed at the proposed slip velocity in this study, with a higher mass flow rate in both the vertical and horizontal directions as compared to the results of the algebraic slip velocity. In summary, it is concluded that the conventional slip velocity may be able to provide good prediction in nanofluid flow for low volume fractions, but implementing the other slip mechanisms is essential with a rise in concentration.

\section{Nomenclature}

\begin{tabular}{l|l|l|l}
$A$ & Hamaker constant $(\mathrm{J})$ & $R a$ & Rayleigh number \\
\hline$C_{L}$ & Lift coefficient & $R e$ & Reynolds number \\
\hline$c_{p}$ & Specific heat $\left(\mathrm{J} / \mathrm{kg} .{ }^{0} \mathrm{~K}\right)$ & $R e_{\omega}$ & vorticity Reynolds number \\
\hline$d$ & Diameter $(\mathrm{m})$ & $T$ & Temperature $(\mathrm{K})$ \\
\hline$d_{c}$ & Water particle diameter $(\mathrm{m})$ & $T_{f r}$ & freezing point $(\mathrm{K})$ \\
\hline$F$ & Faraday constant $\left(\mathrm{C} \mathrm{mol}^{-1}\right)$ & $V_{k m}, V_{p m}$ & Drift velocity $(\mathrm{m} / \mathrm{s})$ \\
\hline$F_{d r a g}$ & drag force $(\mathrm{N} / \mathrm{kg})$ & $V_{R}$ & Potential energy $(\mathrm{J})$ \\
\hline$F_{E D L}$ & $\begin{array}{l}\text { electric double layer } \\
\text { force }(\mathrm{N} / \mathrm{kg})\end{array}$ & $V_{\text {slip }}$ & Slip or relative velocity $(\mathrm{m} / \mathrm{s})$ \\
\hline$F_{\text {lift }}$ & lift force $(\mathrm{N} / \mathrm{kg})$ & $\begin{array}{l}\text { Special } \\
\text { characters }\end{array}$ & $\begin{array}{l}\text { Thermal expansion coefficient } \\
\left(\mathrm{K}^{-1}\right)\end{array}$ \\
\hline$F_{V D W}$ & Van der Waals force $(\mathrm{N} / \mathrm{kg})$ & $\alpha$ & Vacuum permittivity $\mathrm{CV}^{-1} \mathrm{~m}^{-1}$ \\
\hline$F_{\text {Pressure }}$ & pressure gradient force $(\mathrm{N} / \mathrm{kg})$ & $\beta$ & Volume fraction \\
\hline$f_{d}$ & Drag function & $\varepsilon_{0}$ &
\end{tabular}




\begin{tabular}{|c|c|c|c|}
\hline$g$ & $\operatorname{Gravity}\left(\mathrm{m} / \mathrm{s}^{2}\right)$ & $\varepsilon_{r}$ & Relative permittivity \\
\hline$H$ & Enthalpy (J/kg) & $\kappa$ & Debye-Huckel parameter $\left(\mathrm{m}^{-1}\right)$ \\
\hline$I_{0}$ & Ionic strength (mol) & $\mu$ & Dynamic viscosity (kg/m.s) \\
\hline$h$ & Particle to particle distance $(\mathrm{m})$ & $v$ & Kinematic viscosity $\left(\mathrm{m}^{2} / \mathrm{s}\right)$ \\
\hline$J_{p}$ & Particle mass flux (kg/m.s) & $\rho$ & Density $\left(\mathrm{kg} / \mathrm{m}^{3}\right)$ \\
\hline$K_{B}$ & $\begin{array}{l}\text { Boltzmann constant } \\
\left(\mathrm{m}^{2} \cdot \mathrm{kg} /{ }^{\circ} \mathrm{K} \cdot \mathrm{s}^{2}\right)\end{array}$ & $\psi$ & Surface potential (V) \\
\hline$k$ & $\begin{array}{l}\text { Thermal conductivity } \\
\left(\mathrm{W} / \mathrm{m} .{ }^{\circ} \mathrm{K}\right)\end{array}$ & $\tau$ & particle relaxation time(s) \\
\hline$L$ & $\begin{array}{l}\text { Cavity characteristic length } \\
\text { (m) }\end{array}$ & $\tau_{m}$ & Mixture shear stress $(\mathrm{Pa})$ \\
\hline$L_{D}$ & double layer thickness(m) & $R e_{\omega}$ & Vorticity Reynolds number \\
\hline $\mathrm{Nu}$ & Nusselt number & Subscripts & \\
\hline$P r$ & Prandtl number & $c$ & Continuous phase \\
\hline$q$ & Conduction heat flux $\left(\mathrm{W} / \mathrm{m}^{3}\right)$ & $m$ & Mixture \\
\hline$R$ & $\begin{array}{l}\text { Gas universal constant }\left(\mathrm{J} \mathrm{mol}^{-1}\right. \\
\left.\mathrm{K}^{-1}\right)\end{array}$ & $p$ & Particle \\
\hline
\end{tabular}

\section{References}

[1] H. Xie, J. Wang, T. Xi, Y. Liu, F. Ai, Q. Wu. Thermal conductivity enhancement of suspensions containing nanosized alumina particles, Journal of Applied Physics 91 (2002) $4568-4572$.

[2] S.P. Jang, S.U.S. Choi. Effects of various parameters on nanofluid thermal conductivity. Journal of Heat Transfer 129 (2007) 617-623.

[3] R. Karthik, R.H. Nagarajan, B. Raja, P. Damodharan. Thermal conductivity of CuO-DI water nanofluids using 3-w measurement technique in a suspended micro-wire, Experimental Thermal and Fluid Science 40 (2012) 1-9.

[4] X. Li, C. Zou, X. Lei, W. Li. Stability and enhanced thermal conductivity of ethylene glycol-based SiC nanofluids, International Journal of Heat and Mass Transfer 89 (2015) 613-619.

[5] W.H. Azmi, K.V. Sharma, R. Mamat, G. Najaf, M.S. Mohamad. The enhancement of effective thermal conductivity and effective dynamic viscosity of nanofluids - a review, Renewable Sustainable Energy Reviews 53 (2016) 1046-1058.

[6] D. Wen, Y. Ding. Formulation of nanofluids for natural convective heat transfer applications, International Journal of Heat and Fluid Flow 26 (2005) 855-864. 
[7] D.K. Agarwal, A. Vaidyanathan, S.S. Kumar. Experimental investigation on thermal performance of kerosene - graphene nanofluid, Experimental Thermal and Fluid Science 71 (2016) 126-137.

[8] S. Halelfadl, P. Estellé, B. Aladag, N. Doner, T. Maré. Viscosity of carbon nanotubes water-based nanofluids: influence of concentration and temperature, International Journal of Thermal Sciences 71 (2013) 111-117.

[9] A.G.A. Nnanna. Experimental model of temperature - driven nanofluid, Journal of Heat Transfer 129 (2007) 697-704.

[10] R. Ni, S.Q. Zhou, K.Q. Xia. An experimental investigation of turbulent thermal convection in waterbased alumina nanofluid, Physics Fluids 23 (2011) 022005.

[11] C.J. Ho, D.S. Chen, W.M. Yan, O. Mahian. Buoyancy-driven flow of nanofluids in a cavity considering the Ludwig-Soret effect and sedimentation: numerical study and experimental validation, International Journal of Heat and Mass Transfer 77 (2014) 684694.

[12] A.K. Tiwari, P. Ghosh, J. Sarkar. Particle concentration levels of various nanofluids in plate heat exchanger for best performance, International Journal of Heat and Mass Transfer 89 (2015) 1110-1118.

[13] C.H. Li, G.P. Peterson. Experimental studies of natural convection heat transfer of $\mathrm{Al}_{2} \mathrm{O}_{3} / \mathrm{DI}$-water nanoparticle suspensions (nanofluids), Advanced Mechanical Engineering 2 (2010) 742739.

[14] D. Liu, L. Yu. 2011. Single-phase thermal transport of nanofluids in a minichannel, Journal of Heat Transfer 133 (2011) 031009.

[15] L.S. Sundar, K.V. Sharma. Turbulent heat transfer and friction factor of $\mathrm{Al}_{2} \mathrm{O}_{3}$ nanofluid in circular tube with twisted tape inserts, International Journal of Heat and Mass Transfer 53 (2010) 1409-1416.

[16] M. Goodarzi, M.R. Safaei, K. Vafai, G. Ahmadi, M. Dahari, S.N. Kazi, N. Jomhari. Investigation of nanofluid mixed convection in a shallow cavity using a two-phase mixture model, International Journal of Thermal Sciences 75 (2014) 204-220.

[17] Y. Xuan, W. Roetzel. Conceptions for heat transfer correlation of nanofluids, International Journal of Heat and Mass Transfer 43 (2000) 3701-3707.

[18] S.E.B Maiga, S.J. Palm, C.T. Nguyen, G. Roy, N. Galanis. 2005. Heat transfer enhancement by using nanofluids in forced convection flows, International Journal of Heat and Fluid Flow 26 (2005) 530-546.

[19] A. Akbarinia, R. Laur. Investigating the diameter of solid particles effects on a laminar nanofluid flow in a curved tube using a two phase approach, International Journal of Heat and Fluid Flow 30 (2009) 706-714.

[20] V. Bianco, O. Manca, S. Nardini. Numerical investigation on nanofluids turbulent convection heat transfer inside a circular tube, International Journal of Thermal Sciences 50 (2011) 341-349. 
[21] M.M. Rashidi, A. Hosseini, I. Pop, S. Kumar, N. Freidoonimehr. Comparative numerical study of single and two-phase models of nanofluid heat transfer in wavy channel, Applied Mathematics and Mechanics (English Edition) 35 (7) (2014) 831-848.

[22] M. Shariat, R.M. Moghari, A. Akbarinia, R. Rafee, S.M. Sajjadi. Impact of nanoparticle mean diameter and the buoyancy force on laminar mixed convection nanofluid flow in an elliptic duct employing two-phase mixture model, International Communications in Heat and Mass Transfer 15 (2014) 15-24.

[23] Y. Abbassi, A.S. Shirani, S. Asgarian. Two-phase mixture simulation of $\mathrm{Al}_{2} \mathrm{O}_{3} /$ water nanofluid heat transfer in a non-uniform heat addition test section, Progress Nuclear Energy 83 (2015) 356-364.

[24] F. Garoosi, B. Rohani, M.M. Rashidi. Two-phase mixture modeling of mixed convection of nanofluids in a square cavity with internal and external heating, Powder Technology 275 (2015) 304-321.

[25] M. Hejazian, M.K. Moraveji, A. Beheshti. Comparative study of Euler and mixture models for turbulent flow of $\mathrm{Al}_{2} \mathrm{O}_{3}$ nanofluid inside a horizontal tube, International Communications in Heat and Mass Transfer 52 (2014) 152-158.

[26] M. Mahdavi, M. Sharifpur, J.P. Meyer. CFD modelling of heat transfer and pressure drops for nanofluids through vertical tubes in laminar flow by Lagrangian and Eulerian approaches, International Journal of Heat and Mass Transfer 88 (2015) 803-813.

[27] J. Buongiorno. 2006. Convective transports in nanofluids, Journal of Heat Transfer 128 (2006) 240-250.

[28] K.S. Hwang, S.P. Jang, S.U.S. Choi. Flow and convective heat transfer characteristics of water-based $\mathrm{Al}_{2} \mathrm{O}_{3}$ nanofluids in fully developed laminar flow regime, International Journal of Heat and Mass Transfer 52 (2009) 193-199.

[29] A.V. Kuznetsov, D.A. Nield. Natural convective boundary-layer flow of a nanofluid past a vertical plate, International Journal of Thermal Sciences 49 (2010) 243-247.

[30] F. Garoosi, L. Jahanshaloo, M.M. Rashidi, A. Badakhsh, M.E. Ali. Comparative Numerical Study of Single-Phase and Two-Phase Models for Bio-Nanofluid Transport Phenomena, Journal of Mechanics in Medicine and Biology 14 (2014) 183-203.

[31] H.A. Pakravan, M. Yaghoubi. Analysis of nanoparticles migration on natural convective heat transfer of nanofluids, International Journal of Thermal Sciences 68 (2013) 79-93.

[32] E.R.D. Schio, M. Celli, A. Barletta. Effects of Brownian diffusion and thermophoresis on the laminar forced convection of a nanofluid in a channel, Journal of Heat Transfer 136 (2014) 022401.

[33] F. Garoosi, S. Garoosi, K. Hooman. Numerical simulation of natural convection and mixed convection of the nanofluid in a square cavity using Buongiorno model, Powder Technology 268 (2014) 279-292.

[34] Z. Haddad, E. Abu-Nada, H.F. Oztop, A. Mataoui. Natural convection in nanofluids: are the thermophoresis and Brownian motion effects significant in nanofluid heat transfer enhancement? International Journal of Thermal Sciences 57 (2012) 152-162. 
[35] M. Manninen, V. Taivassalo, S. Kallio. On the mixture model for multiphase flow. Technical Research Centre of Finland, VTT Publications 288.

[36] J.S. Marshall, S. Li. Adhesive particle flow, in a discrete-element approach, first edition. Cambridge: Cambridge University Press.

[37] D.A. Drew, R.T. Lahey. 1993. Particulate two-phase flow. Boston, MA: ButterworthHeinemann.

[38] P.G. Saffman. The lift on a small sphere in a slow shear flow, Journal of Fluid Mechanics 22 (1965) 385-400.

[39] R.J. Hunter. 2001. Foundations of colloid science. Oxford: Oxford University Press.

[40] American Society of Heating, Refrigerating and Air-conditioning Engineers (ASHRAE). 2009. Fundamentals. Appendix A. Atlants, GA: ASHRAE.

[41] M. Corcione. Empirical correlating equations for predicting the effective thermal conductivity and dynamic viscosity of nanofluids, Energy Conversion and Management 52 (2011) 789-793.

[42] W. Wu, D. Ewing, C.Y. Ching. Investigation of a large top wall temperature on the natural convection plume along a heated vertical wall in a square cavity, International Journal of Heat and Mass Transfer 51 (2008) 1551-1561.

[43] C.J. Ho, W.K. Liu, Y.S. Chang, C.C. Lin. Natural convection heat transfer of Aluminawater nanofluid in vertical square enclosures: an experimental study, International Journal of Thermal Sciences 49 (2010) 1345-1353. 
Table 1: Comparative study of slip velocities induced by forces

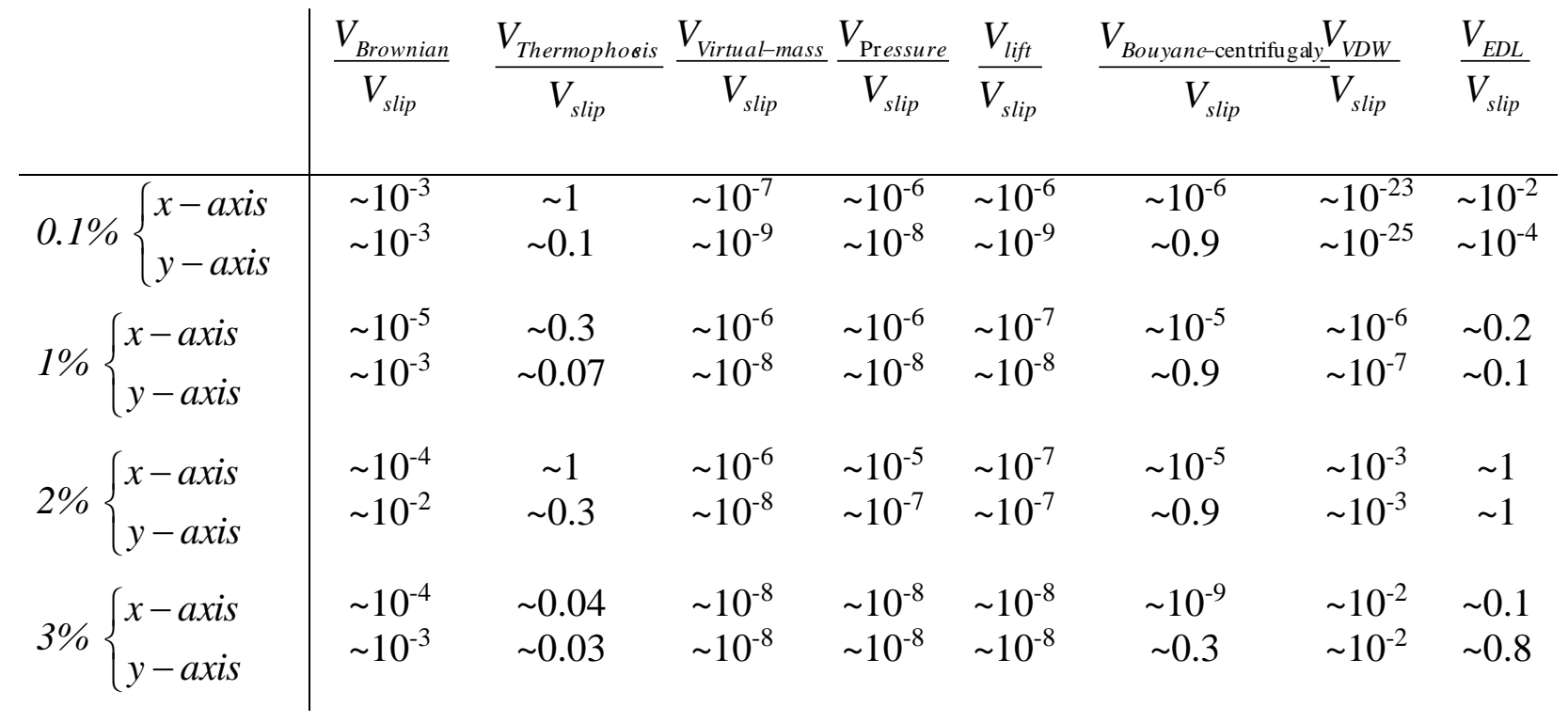




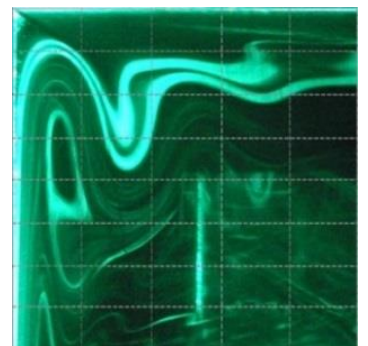

Experimental [42]

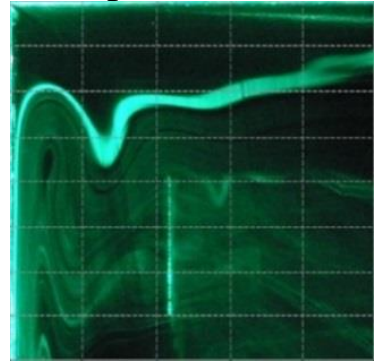

Experimental [42]

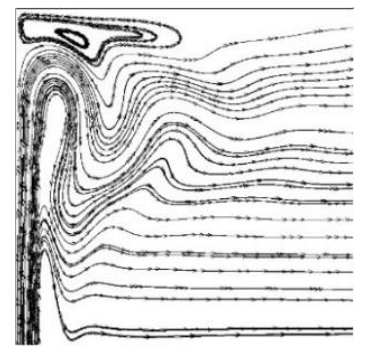

Numerical

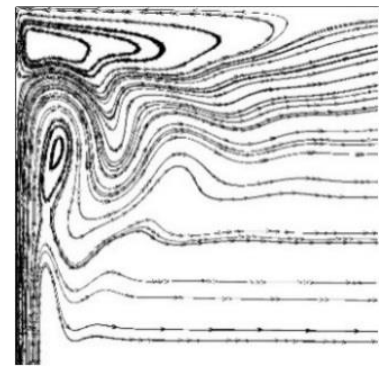

Numerical

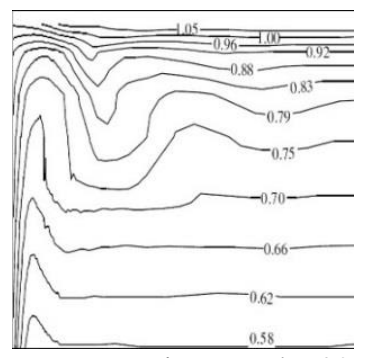

Experimental [42]

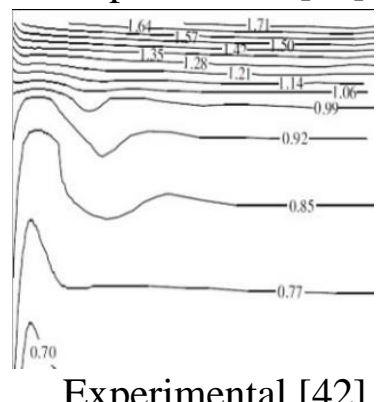

Experimental [42]

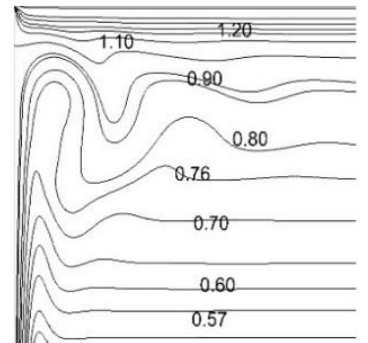

Numerical

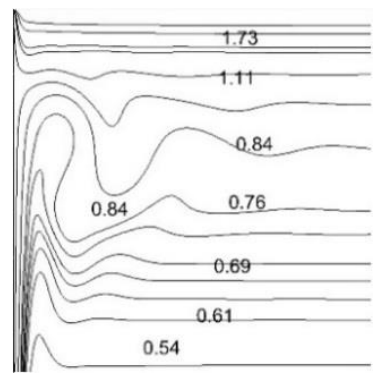

Numerical

Figure 1: Comparison of experimental flow pattern and non-dimensional temperature with numerical results. The first row is the Grashof number $(\mathrm{Gr})=1.39 \times 10^{8}$ and the second row is $\mathrm{Gr}=1.44 \times 10^{8}$ 


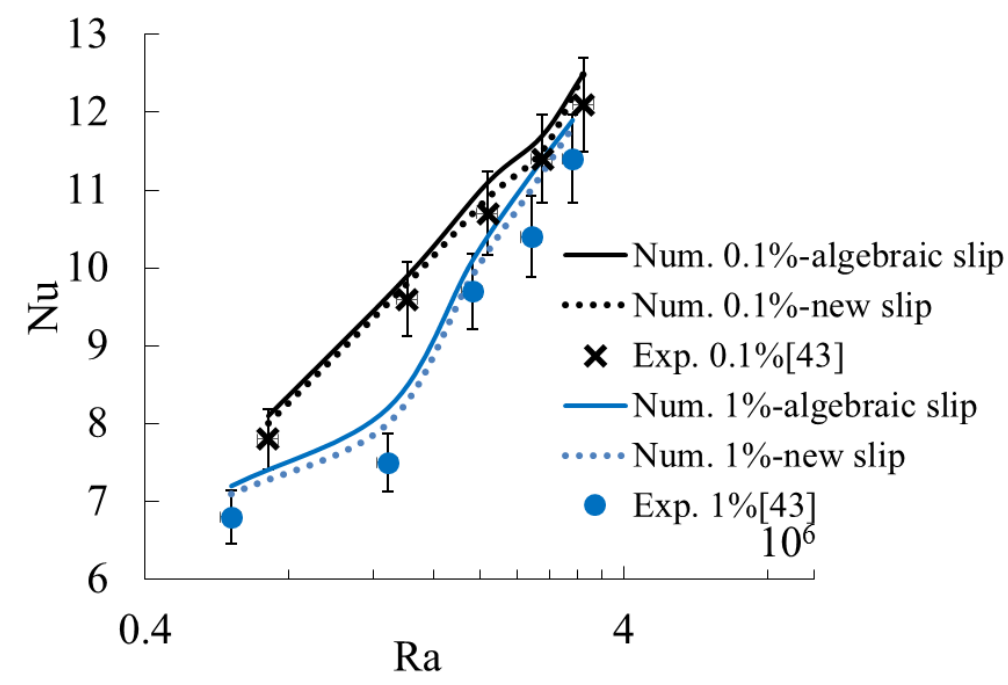

a)

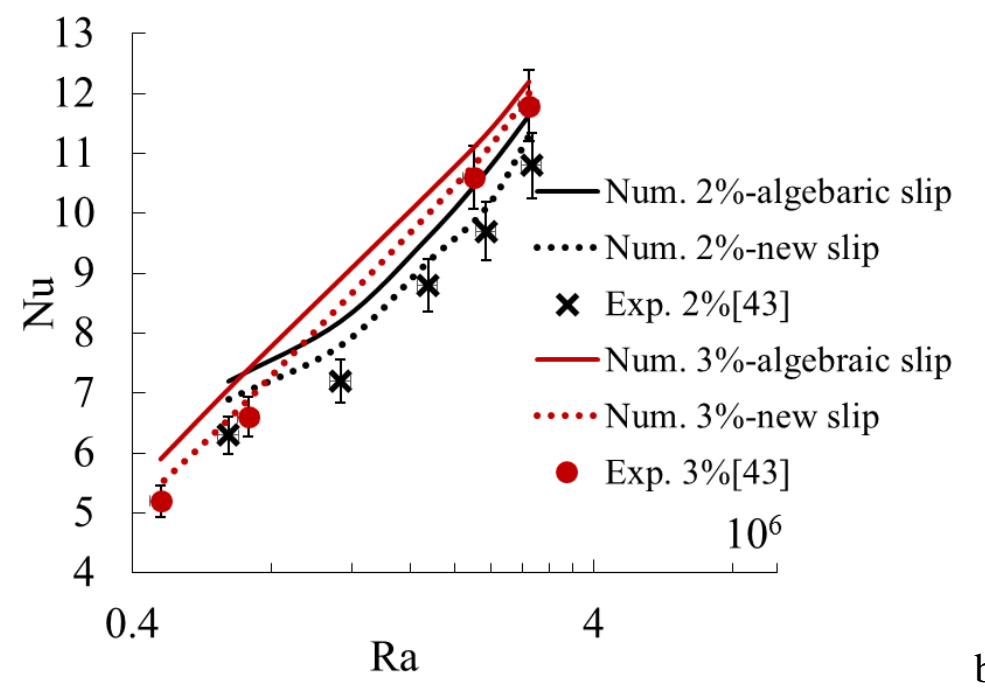

Figure 2: Comparing the Nusselt number estimated by the conventional algebraic and the proposed slip velocity with the experimental measurements for alumina nanofluid 

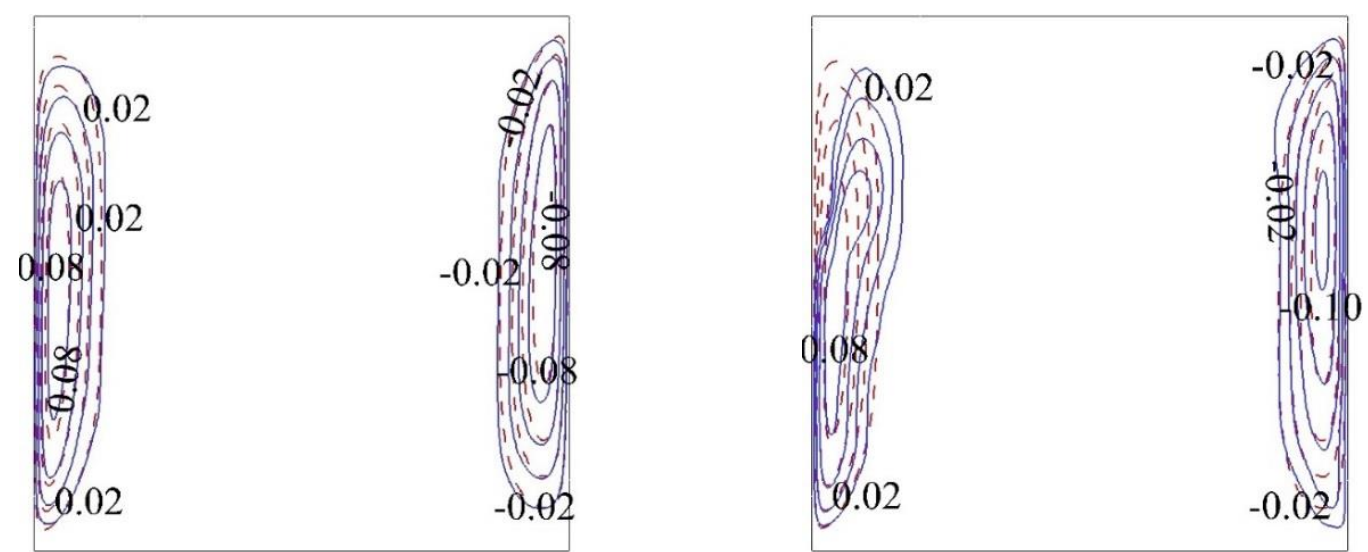

a) $3 \%$ - Algebraic slip velocity

b) $3 \%-$ New slip velocity
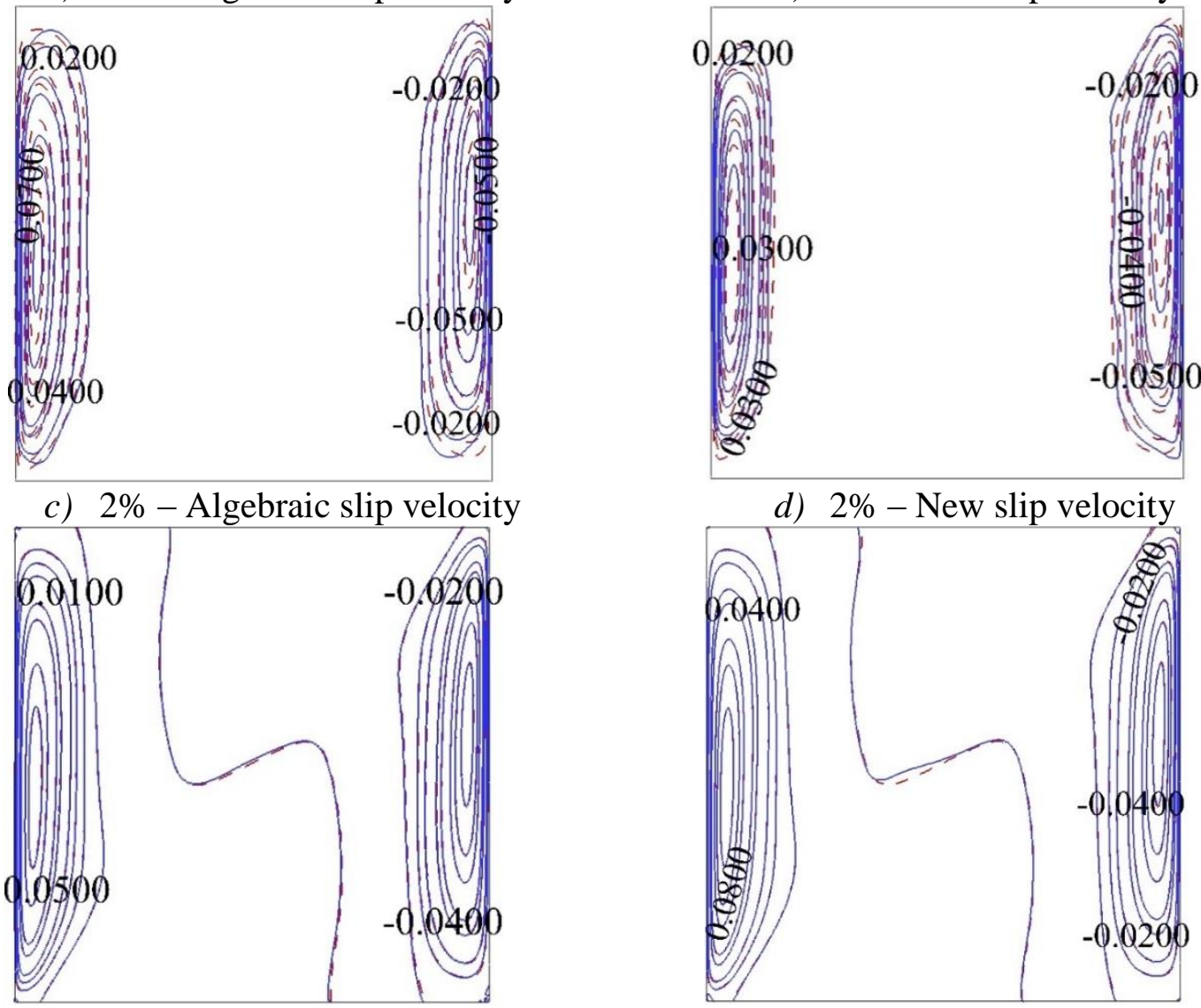

e) $0.1 \%$ - Algebraic slip velocity

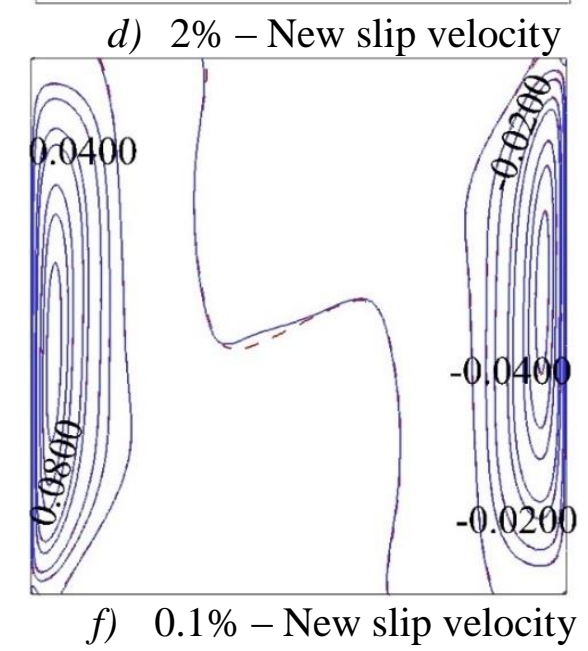

Figure 3: Non-dimensional flow velocity in the Y-direction for water (dashed red lines) and for alumina nanofluid (blue solid lines) with algebraic and new slip velocities 


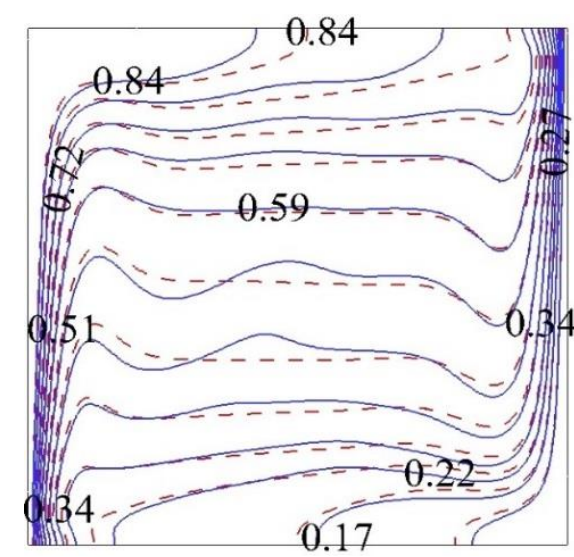

a) $3 \%$ - Algebraic slip velocity

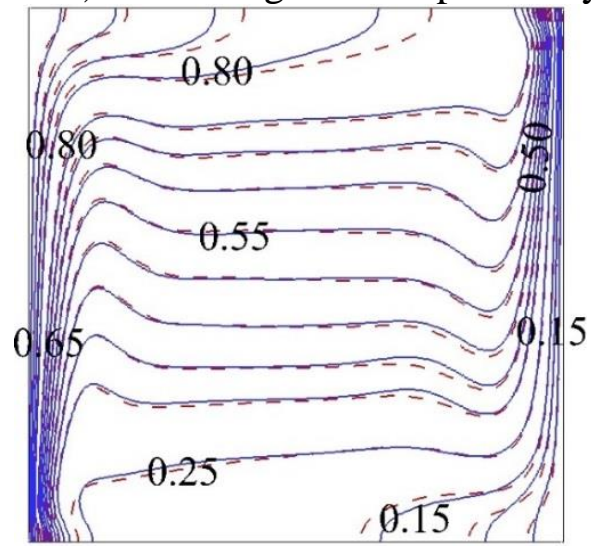

c) $2 \%$ - Algebraic slip velocity

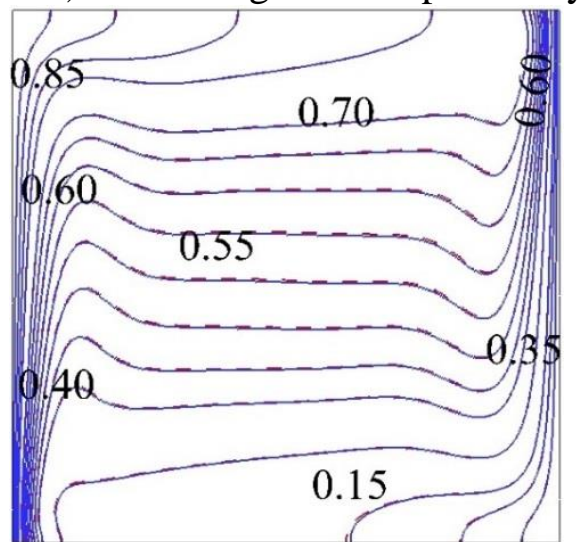

e) $0.1 \%$ - Algebraic slip velocity

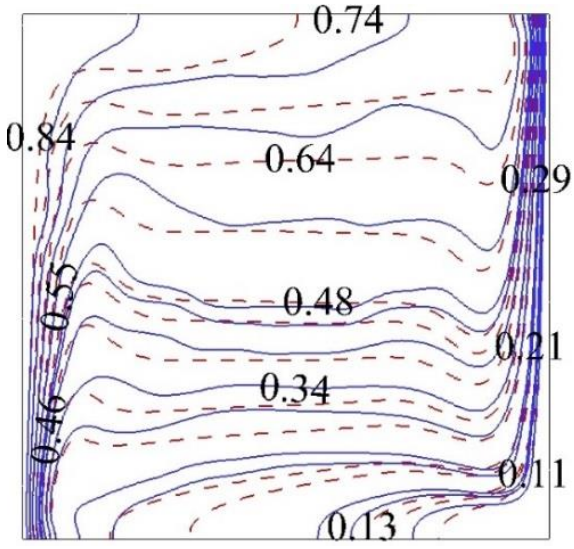

b) $3 \%$ - New slip velocity

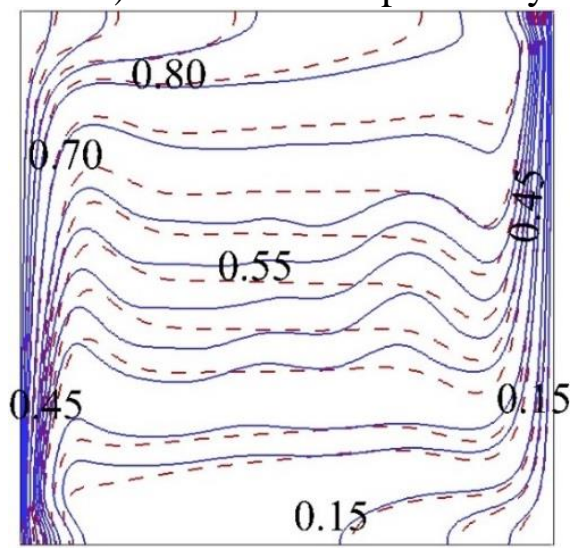

d) $2 \%-$ New slip velocity

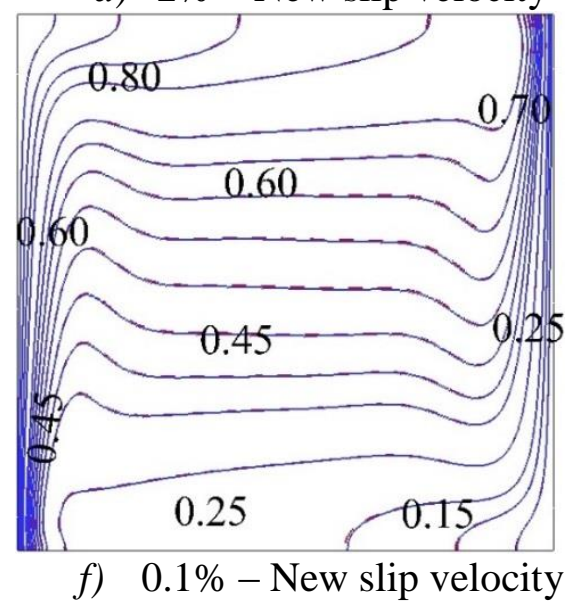

Figure 4: Non-dimensional temperature inside a cavity filled with water (dashed red lines) and alumina nanofluid (solid blue lines) with algebraic and new slip velocities 

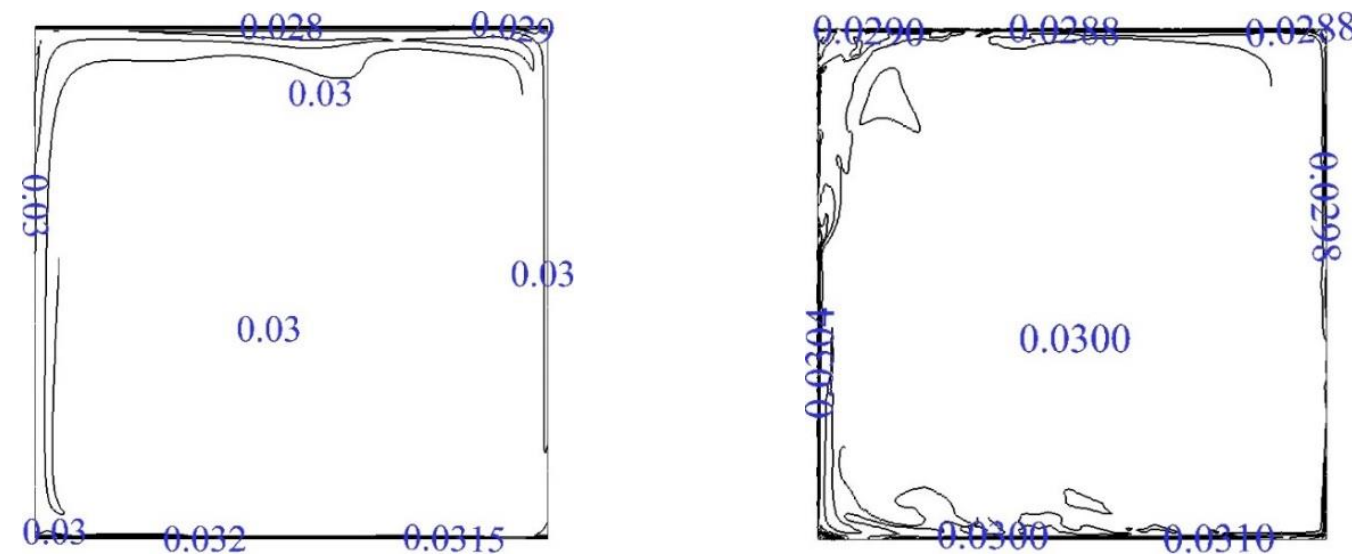

a) $3 \%$ - Algebraic slip velocity

b) $3 \%-$ New slip velocity
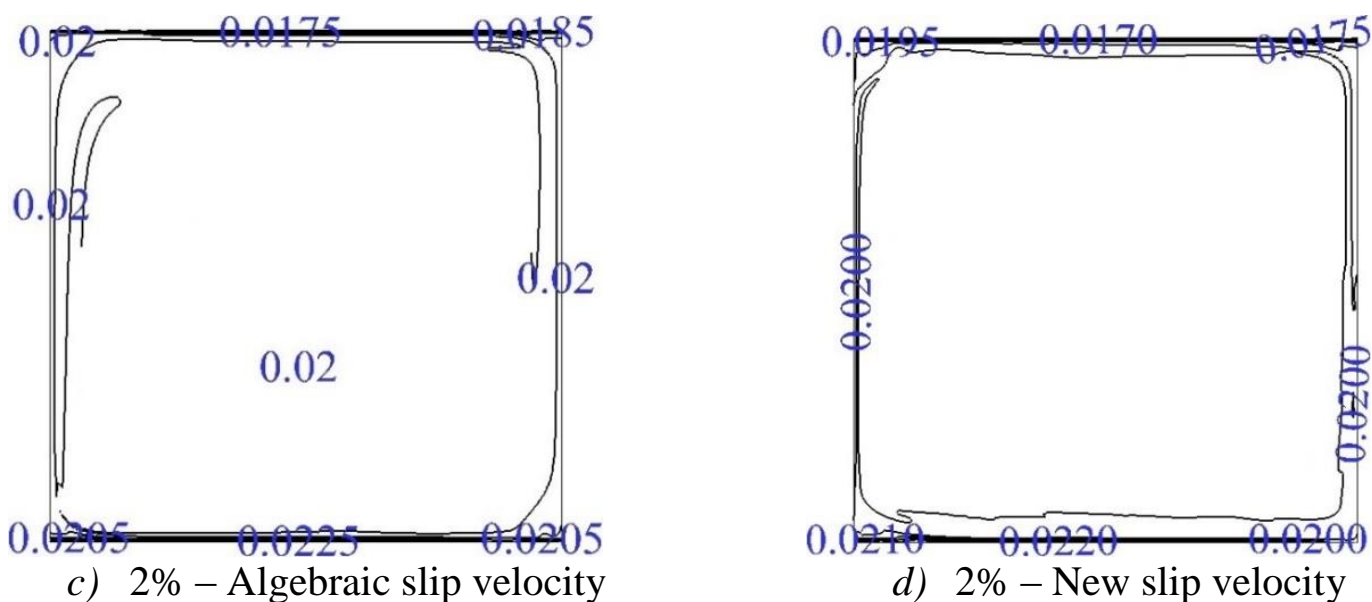

d) $2 \%$ - New slip velocity

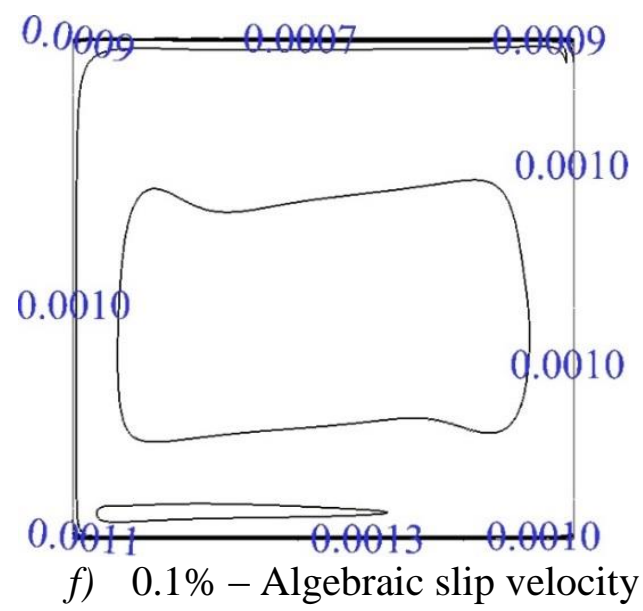

e)

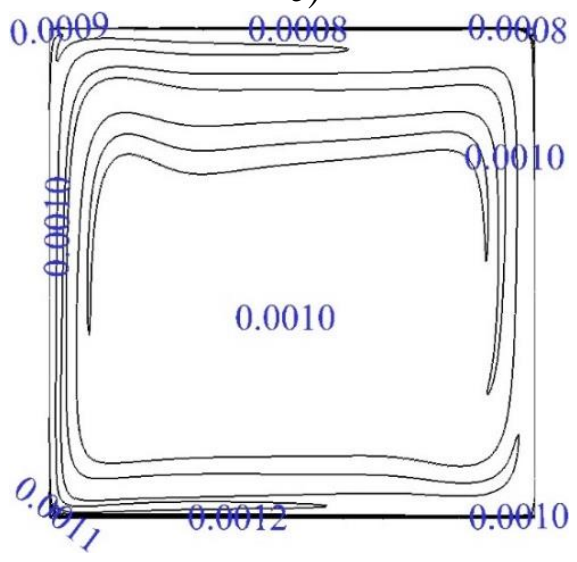

g) $0.1 \%$ - New slip velocity

Figure 5: The distribution of the alumina nanoparticle concentration inside a cavity predicted with the assumption algebraic and new slip velocities 


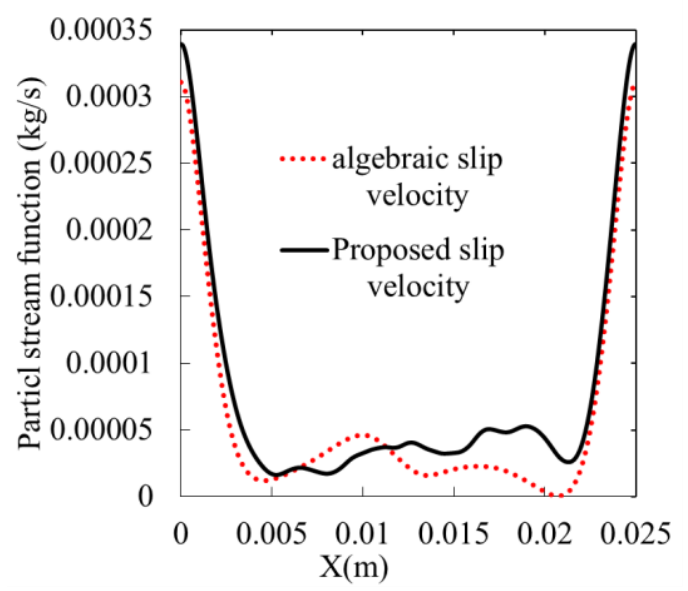

a) A 3\% alumina nanofluid

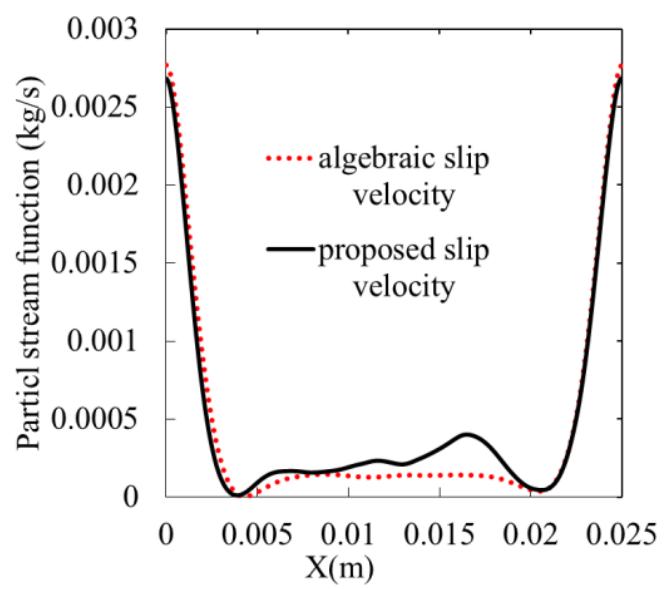

c) A $2 \%$ alumina nanofluid

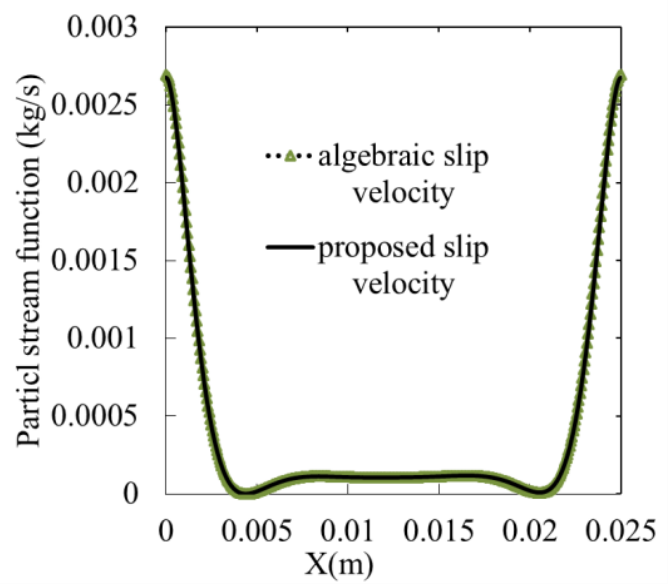

e) A $0.1 \%$ alumina nanofluid

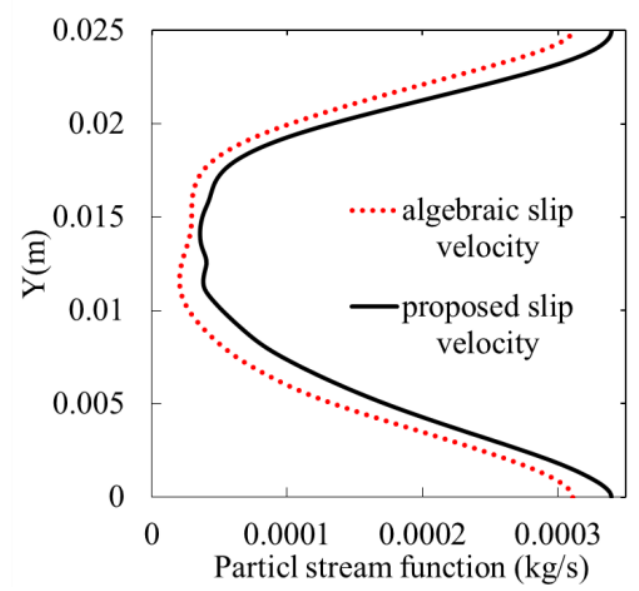

b) A $3 \%$ alumina nanofluid

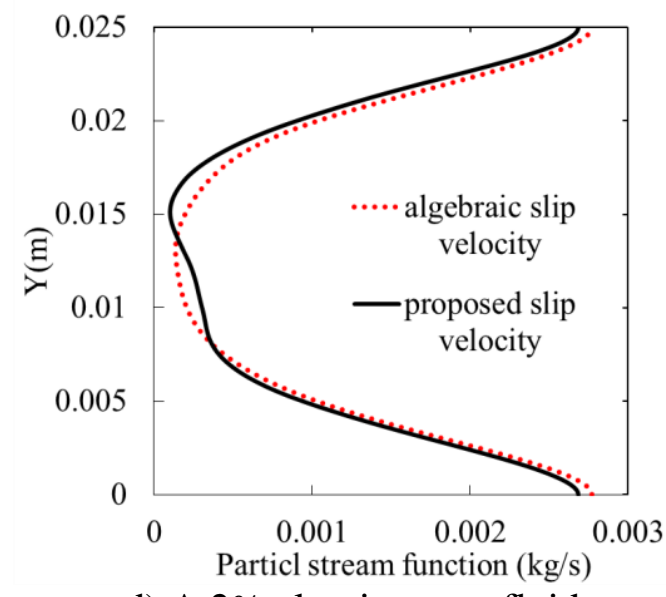

d) A $2 \%$ alumina nanofluid

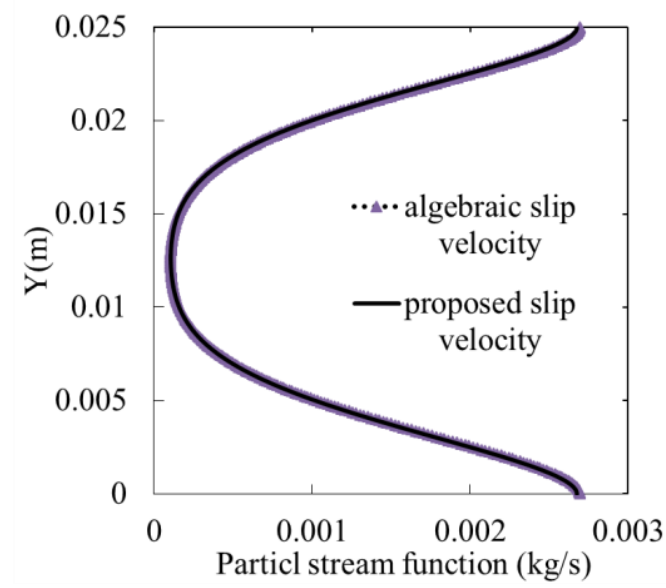

f) A $0.1 \%$ alumina nanofluid

Figure 6: Distribution of particle mass flow rate in the $\mathrm{X}$-direction (from the middle of the hot to the cold wall) and the Y-direction (between the horizontal insulated walls) 


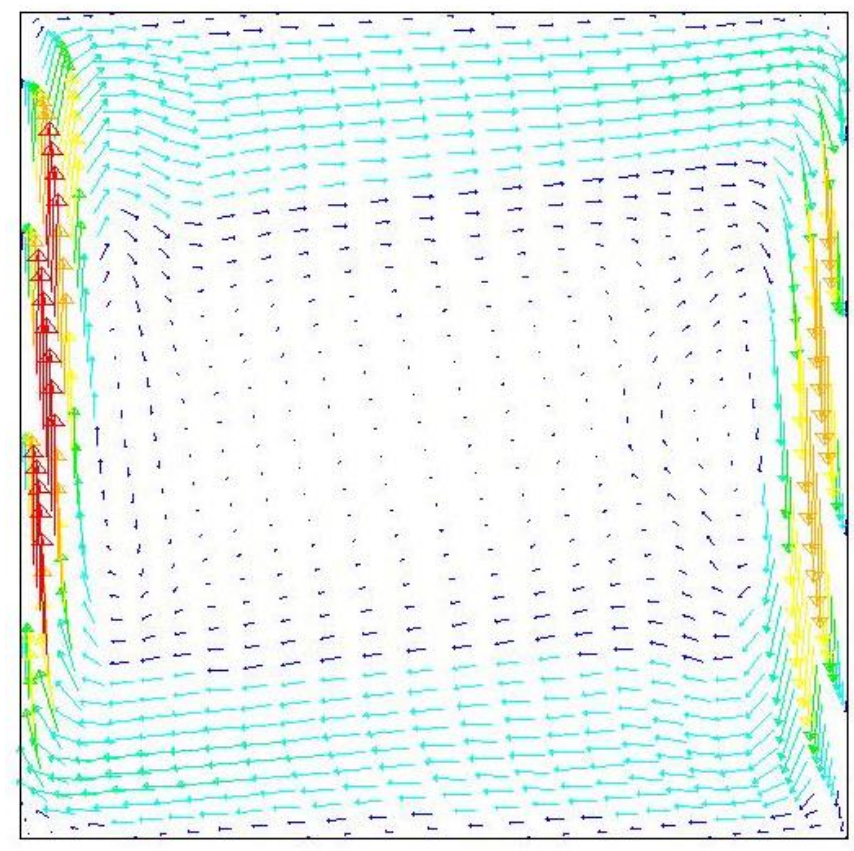

$\mathrm{V}(\mathrm{m} / \mathrm{s})$

Figure 7. Velocity vector inside the cavity coloured by velocity magnitude for alumina nanofluid $2 \%$ vol. 


\section{Biographic information}

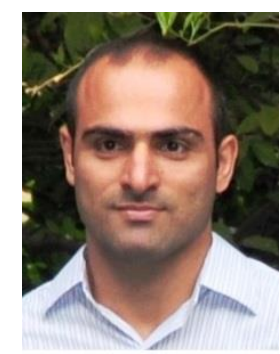

Mostafa Mahdavi is a PhD candidate in the Department of Mechanical and Aeronautical Engineering at the University of Pretoria. He received his master's degree from Shiraz University in Iran. His research interests include heat and mass transfer, natural and force convection, turbulence, nanofluids, particle migration and multiphase flow modelling.

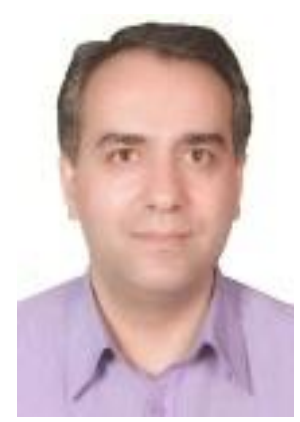

Mohsen Sharifpur is a senior lecturer in the Department of Mechanical and Aeronautical Engineering at the University of Pretoria and is responsible for the Nanofluids Research Laboratory. He received a BEng (Mechanical Engineering) degree from Shiraz University in Iran. He completed a MEng degree in nuclear engineering (thermal fluid) and received a full scholarship for his $\mathrm{PhD}$ study in mechanical engineering (thermal fluid) from the Eastern Mediterranean University. He was the only postgraduate student who received four out of four for the cumulative grade point average (CGPA) when he obtained his $\mathrm{PhD}$. He is also registered as a professional engineer. He is the author and co-author of more than 70 articles and conference papers. His research interests include convective multiphase flow, the thermal fluid behaviour of nanofluids, convection nanofluids, convection in porous media, computational fluid dynamics (CFD) and waste heat to work in thermal systems. He also reviews notable accredited journals.

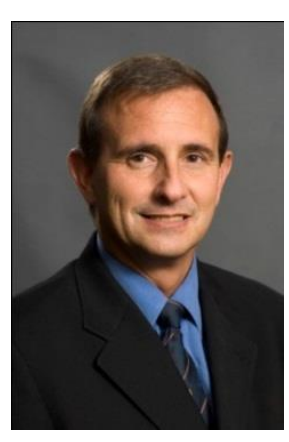

Josua Meyer obtained his BEng degree cum laude in 1984, an MEng degree cum laude in 1986 and a $\mathrm{PhD}$ in 1988, all in mechanical engineering from the University of Pretoria. He is registered as a professional engineer. After his military service from 1988 to 1989 , he accepted a position as associate professor in the Department of Mechanical Engineering at the erstwhile Potchefstroom University for Christian Higher Education (now North-West University) in 1990. He was acting head and professor in this department until he accepted a position as professor in the Department of Mechanical and Manufacturing Engineering at the erstwhile Rand Afrikaans University (now the University of Johannesburg) in 1994. He was Chairman of the Department from 1999 until the end of June 2002, when he was appointed Professor and Head of the Department of Mechanical and Aeronautical Engineering at the University of Pretoria. Presently, he is the Chair of the School of Engineering at the University of Pretoria. He specialises in heat transfer, fluid mechanics and the thermodynamic aspects of heating, ventilation and air-conditioning. He is the author and co-author of more than 500 articles, conference papers and patents and has received various prestigious awards for his research. $\mathrm{He}$ is also a fellow or member of various professional institutes and societies, such as the South African Institute for Mechanical Engineers, the South African Institute for Refrigeration and Air-conditioning, the American Society for Mechanical Engineers and the American Society of Heating, Refrigeration and Air-conditioning Engineers. He is regularly 
invited as a keynote speaker at local and international conferences. He has received various teaching and exceptional achiever awards. He is an associate editor of Heat Transfer Engineering and editor of Journal of Porous Media 